\title{
Interdependencia entre Educación y Conservación en los Museos de Arte Contemporáneo
}

\section{Flavia Parisia ${ }^{\mathrm{a}}$, Dr. Rosario Llamas Pacheco ${ }^{\mathrm{b}}$}

${ }^{a}$ Candidata a doctor, Universitat Politècnica de València, Spain, flaviaparisi1@gmail.com; ${ }^{\text {PProfesora, }}$ Universitat Politècnica de València, Spain.

\section{Resumen}

El presente trabajo se enmarca dentro de un proyecto de doctorado de la Universitat Politècnica de València, enfocado en el estudio de la interdependencia entre cuestiones de educación y de conservación en los museos de arte contemporáneo.

A partir de un enfoque hacia la compleja relación entre el público y las obras de arte contemporáneo, la investigación propone casos de estudio en los cuales la colaboración entre distintos sectores de los museos de arte contemporáneo, en particular los de educación y de conservación, puede estimular un sentido de apropiación en el público que derive en una mayor preocupación por el futuro de su patrimonio contemporáneo.

En este estudio se proponen tres proyectos en los cuales el público aprende las funciones del museo, recibiendo pequeñas tareas como por ejemplo simular la selección, manejo, transporte y exposición de las obras. Además, se incluye un proyecto en el cual el público tiene la posibilidad de aprender cómo interactuar con algunas obras originales, descubriendo su complejidad material y estética a través del tacto.

Estas experiencias ofrecen tanto un conocimiento de las características técnicas y materiales de los objetos, lo cual se está perdiendo rápidamente en la sociedad contemporánea, a la vez que muestran un cambio en la institución museística hacia una corresponsabilidad entre profesionales y público en el cuidado del patrimonio contemporáneo.

Palabras clave: educación, conservación, museos de arte contemporáneo 


\section{Introducción}

Las colecciones de arte contemporáneo plantean muchos desafíos conservativos, y entre ellos, la necesidad de interacción con el público como uno de los más importantes. Estos desafíos empiezan con la dificultad del público para comprender las obras de arte contemporáneo y también para compreder la naturaleza de los museos que las exponen. Desde las ambigüedades cronológicas relativas al arco de tiempo en el cual se inscribe la producción artística denominada contemporánea, hasta las ambigüedades expositivas sobre las obras con las cuales se puede interactuar físicamente y en las cuales el contacto no está permitido, el público se encuentra frente a colecciones complejas, tanto en la variedad de los contenidos, como de los materiales y las técnicas. La museografía de los museos de arte contemporáneo no ayuda el público en la comprensión de estas colecciones, ya que incluye experiencias muy variadas, desde las visitas tradicionales en las cuales no hay contacto con la obra, hasta configuraciones inmersitas que rompen con los esquemas museográficos más familiares para el público. La arquitectura también juega un papel interesante al proponer al visitante colecciones de arte contemporáneo exhibidas en edificios históricos, en edificios industriales readaptados, y en nuevas construcciones de tipo escultórico, frecuentemente realizadas por arquitectos estrellas. Además, un relativismo interpretativo general autoriza cualquier tipo de especulación estética, y estimula al público a realizar un juicio de valor sobre estas colecciones, que parece frecuentemente más bajo que en otras colecciones patrimoniales. Las obras de arte contemporáneo, incluso cuando se trata de obras de más de 40 o 50 años, parecen pasar a un segundo lugar por no tener el peso de la historia, y por ser de difícil comprensión, tanto en su identidad artística como en los contextos que las rodean. Así, cuando el público tiene la posibilidad de interactuar con este tipo de colecciones, lo hace de una forma que parece más parecida a la de un parque de atracciones que a la de un museo. El público interactúa con las obras, tanto si son interactivas como si no, en una modalidad a veces posesiva y no siempre conectiva o explicativa. 


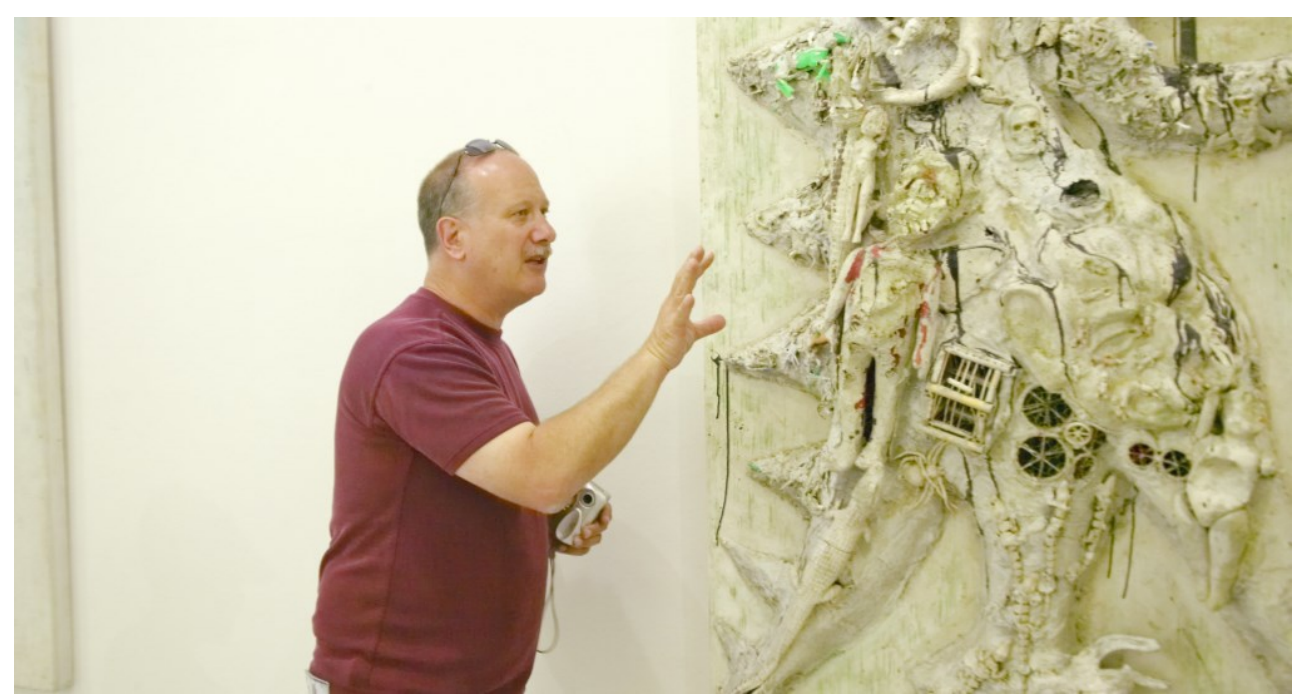

Fig. 1 Turista en el Museo de Arte Moderno y Contemporaneo de Niza, Francia. (C) Americanspirit

\section{Objetivos}

¿Cómo encontrar un equilibrio entre las necesidades conservativas y las necesidades de interacción de las obras, para que el Museo cumpla con su misión educativa en una perspectiva a largo plazo?

El proyecto de investigación doctoral propone responder a esta pregunta a través de una serie de casos de estudio que destacan la necesidad de colaboración entre distintos sectores del museo para que los visitantes sean enriquecidos por una interacción con las obras que sea completa, incluyendo un conocimiento empírico de los materiales, las técnicas, y las biografías de las obras, sin comprometer su supervivencia a largo plazo.

Los tres proyectos presentados en este artículo, una propuesta para el el GUGGENHEIM de Venecia, del MAXXI de Roma y del MUSEION de Bolzano, presentan oportunidades para una interacción informada tanto sobre contenidos, como sobre las tareas del museo sobre preservación y valoración de estas colecciones. El estudio de estos proyectos pretende valorar sus aspectos comunes y potencialidades en la línea de investigación doctoral sobre la interdependencia entre conservación y educación. Además, se propone destacar las dificultades encontradas en las experiencias de las tres instituciones italianas, y determinar los aspectos a mejorar en la implementación de proyectos de este tipo. En fin, se ofrece la oportunidad de acercar los sectores de educación y de conservación de los museos, los cuales en muchos casos difícilmente encuentran compromisos entre sus objetivos; para el 
conservador, el objetivo principal es la preservación de los materiales y de las técnicas en cuanto constituyen los elementos que llevan el mensaje. En su perspectiva, la interacción directa de los visitantes podría determinar varios riesgos para la obra. Para el mediador, en cambio, el enfoque está generalmente en el mensaje, que el visitante logra alcanzar a través de su interacción con la obra. El nuevo enfoque interdisciplinar entre estos dos sectores que se propone con este estudio, apunta a subrayar los beneficios de esta colaboración.

\section{Metodología}

Para cumplir con los objetivos planteados se ha desarrollado un trabajo de campo, a través de visitas a los museos en cuestión, de participación directa (cuando ha sido posible) en algunas de las actividades de los proyectos analizados, y de grabación de entrevistas con los profesionales de educación y conservación del GUGGHENHEIM, del MAXXI y del MUSEION. Las entrevistas han sido distintas la una de la otra, tanto en su modalidad, en cuanto se han realizado a través de ocasiones estructuradas de forma muy diferente, como en las preguntas, las cuales han sido adaptadas a cada caso de estudio para obtener más informaciones. Sin embargo, algunas preguntas han sido comunes a las tres entrevistas, con el objetivo de comparar los aspectos relativos a las colaboraciones entre colegas de distintos sectores, en particular el de la educación y el de la conservación. La visita al Guggenheim de Venecia ha sido realizada en conjunto con la participación en una jornada del proyecto Doppio Senso, seguida por la entrevista a la curadora del proyecto y mediatora cultural, junto con el artista involucrado en el proyecto. En cambio, la entrevista en el MAXXI ha sido posible gracias a una colaboración de 4 meses con el museo en el 2017, y se ha realizado en distintos encuentros con varios profesionales de la institución romana. Por último, la entrevista con el Museion de Bolzano ha sido programada ad hoc tras el conocimiento del proyecto Museion: Chi Dove Cosa, que presentaba aspectos comunes con el del MAXXI.

El trabajo de campo ha sido acompañado por el análisis de fuentes bibliográficas y de material informativo sobre los respectivos proyectos logrados también a través de las entrevistas. 


\section{Casos de Estudio}

\subsection{El proyecto "Doppio Senso, Percorsi tattili alla Collezione Peggy Guggenheim"1, Museo Guggenheim, Venecia}

El proyecto Doppio Senso, Percorsi tattili alla Collezione Peggy Guggenheim, propone la fruición táctil de una selección tanto de reproducciones de obras, como de obras originales destinado tanto a invidentes y personas con deficiencias visuales, como a videntes. Las piezas varían de un mínimo de 3 hasta un máximo de 6, dependiendo de su complejidad, y accesibilidad. La selección de las obras es efectuada en estrecha colaboración entre el conservador en jefe del Guggenheim, Luciano Pensabene, y la curadora del proyecto Valeria Bottalico: juntos establecen finalidades comunes y las comparten con todo el equipo del museo, para ofrecer la máxima interacción del público con la colección.

El camino educativo tiene el objetivo de difundir el conocimiento del arte moderno y contemporáneo a través de un enfoque en la colección permanente del museo, con análisis de artistas específicos o sobre movimientos artísticos. La exploración táctil, y en particular la actitud con la cual se relacionan con las obras las personas invidentes, puede ofrecer muchas oportunidades educativas incluso para las personas videntes. Las personas videntes generalmente procesan las imágenes de las obras en modo sintético: con una mirada pueden ver las obras en su aparente totalidad, y cuando tienen la posibilidad de tocarlas lo hacen frecuentemente para apropiarse de ellas por un instante o para recibir una confirmación de lo que han visto. Las personas invidentes, en cambio, tocan las obras haciendo con sus manos un recorrido preciso, que los ayuda a construir una imagen mental a partir de una modalidad cognitiva exploratoria, en la cual los particulares de la obra juegan un papel fundamental. Su recorrido, para ser eficaz, necesita ser delicado y necesita de tiempo, un tiempo que el público vidente parece siempre dejar al margen de la experiencia estética. En la presente investigación se plantea la necesidad de formación del público vidente en su interacción con las obras, y se propone la experiencia del publico invidente como estímulo significativo en este camino de formación. Un mejor conocimiento por parte del público de las características materiales de las obras podría reducir las consecuencias de una interacción agresiva, incluso el deterioro de algunas obras, y ofrecer a los visitantes un conocimiento más profundo y completo de la investigación creativa del artista, aprendiendo un respeto hacia la materia que hoy, en muchos casos, se desconoce.

\footnotetext{
1 “Doble sentido, Recorridos Táctiles en la Collación Peggy Guggenheim" (traducción por la autora).
} 

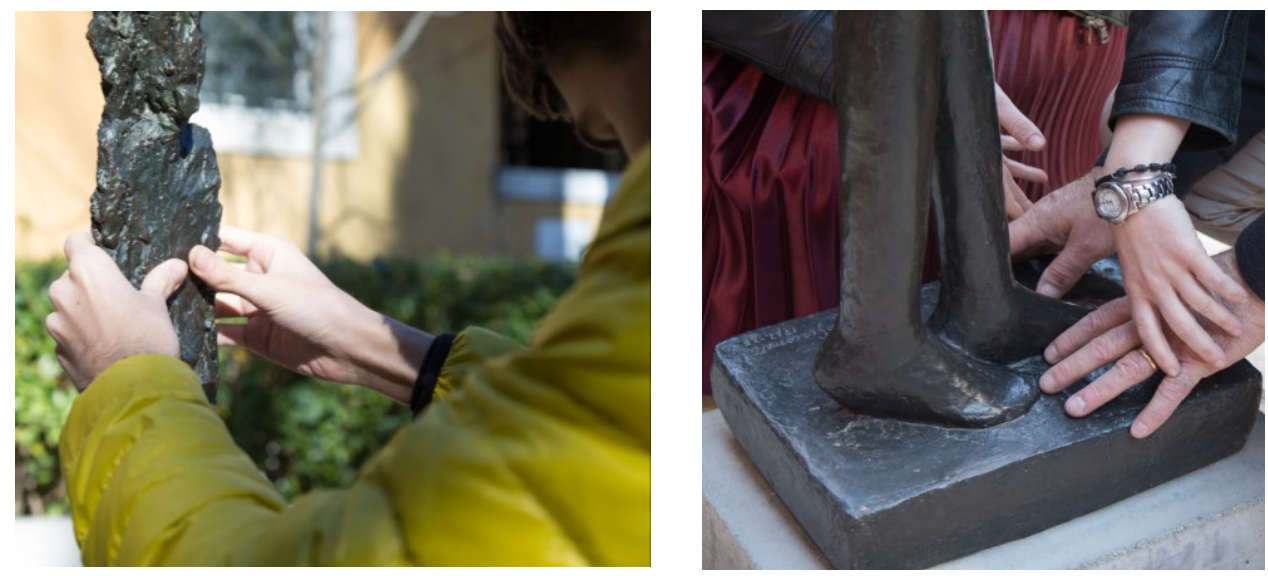

Fig. 2 Exploracion Tactil de una obras en bronce, de Alberto Giacometti, expuestas en el jardin del Museo Guggenheim,Venecia, @Valeria Bottalico

\subsection{El proyecto "EI Museo va a la escuela" - Museo MAXXI, Roma}

En la edición del 2015 del proyecto del MAXXI, Museo Nacional de las Artes del siglo XXI de Roma se han instalado tres obras seleccionas de la colección permanente del Museo (Iran Do Espirito Santo, Extension/Fade, 2005-2006 | Bernard Khoury, Derailing Beirut, 2010 | Jordi Bernadò, tres fotografías, 2007) en los pasillos de tres distintos colegios romanos (Instituto"Virginia Woolf", Instituto "Largo Castelseprio" e Instituto "Roberto Rossellini”). Para la exposición de las obras en las escuelas, el proyecto proponía la colaboración directa con los estudiantes, los cuales han sido formados y responsabilizados a través de visitas a los depósitos del Museo y el laboratorio de conservación, y sesiones con los colegas del Departamento de Manejo de Colecciones y Conservación. En estos encuentros han aprendido algunos de los desafíos relativos a la conservación y exhibición de las obras, incluyendo aspectos de almacenamiento, embalaje, transporte, montaje y mantenimiento. Posteriormente cada clase de estudiantes ha sido encargada de diversas funciones para el mantenimiento de las obras en el tiempo de exposición en las escuelas. Algunos habían recibido la tarea de hacer turnos de guardia cerca de las obras durante las pausas de recreo, otros, de mantener las vitrinas limpias y anotar diariamente el estado de conservación de las obras en un cuaderno, y otros de explicar a los otros estudiantes de la escuela el significado de las obras expuestas. El proyecto ha concluido con la organización de una exposición en la escuela, con reinterpretaciones de las obras realizadas por los estudiantes. 
Entre los objetivos principales del proyecto educativo se destacan los de promover el conocimiento del arte contemporáneo; acercar a los estudiantes a sus familias y a los profesores al museo; crear procesos fructíferos de intercambio, formación y actualización entre los profesionales del museo y el mundo de la escuela; promover la interacción de los estudiantes con actores distintos, como por ejemplo los artistas y los profesionales del museo y del mundo del arte; y activar procesos de aprendizaje informal centrados en la creatividad, pensamiento crítico y conocimiento del contexto territorial.

Para la realización del proyecto el Departamento de Educación, quien ha ideado toda la propuesta, ha trabajado en estrecha colaboración con el Departamento de Manejo de Colecciones, Registro y Conservación, involucrando también a otros sectores del museo. La instalación implicaba varias cuestiones, desde la selección de obras que pudieran ser trasladadas sin demasiados riesgos, hasta la autorización de los artistas, y el seguro de las obras. Este esfuerzo colectivo del museo ha permitido a los estudiantes entrar en el mundo del arte a través de las puertas del Museo como “iniciados”, obteniendo experiencias y nuevos conocimientos. La presencia de las obras en la escuela les ha permitido tener una experiencia de contacto cotidiana, con la posibilidad de tomarse el tiempo necesario para observarlas, entenderlas, y hacerlas propias. Al mismo tiempo los jóvenes han tenido la posibilidad de actuar como propietarios efectivos de su patrimonio contemporáneo, empezando a percibir el Museo como un espacio propio de la comunidad antes que de la institución.

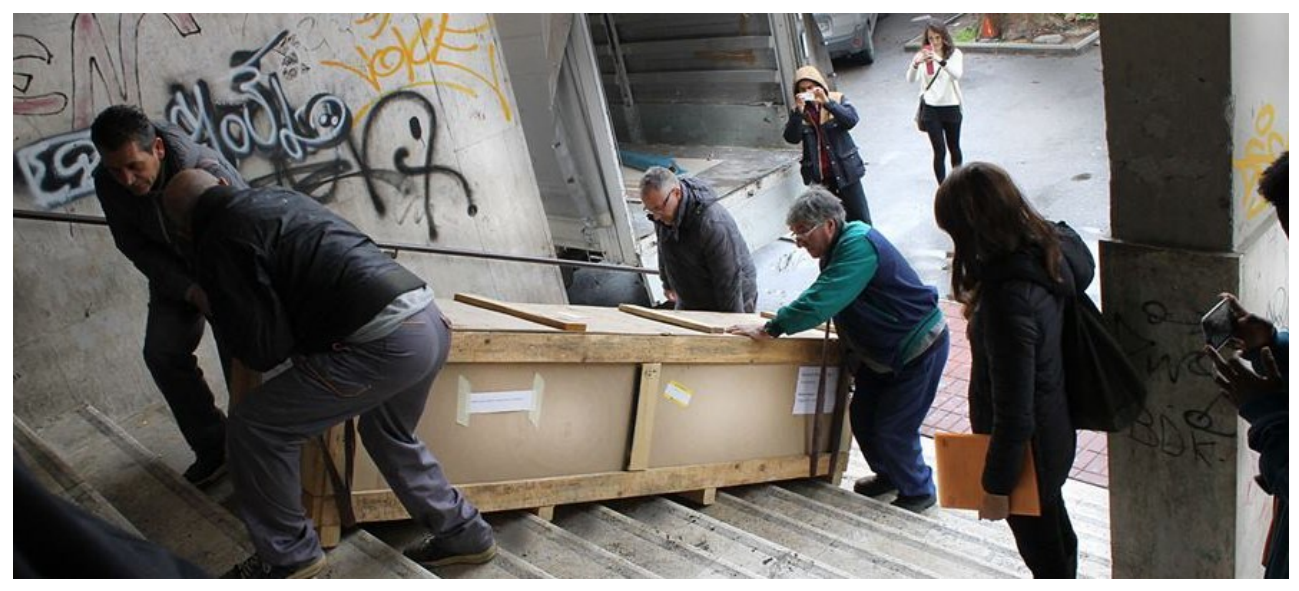

Fig. 3 Transporte de la obra de Bernard Khoury al Instituto ISS "Roberto Rossellini", Photo@Flavio Catuara 
Así, esta edición del Museo va a la escuela propone una nueva imagen del Museo que, compartiendo sus tesoros fuera de sus sedes canónicas, crea una dinámica de intercambio concreto con el público presente en el territorio y alcanza también públicos nuevos en áreas generalmente ajenas al contexto museístico. El Museo pasa de ser un Museo abierto a ser un Museo co-creado, promoviendo la participación y la apropiación del patrimonio por parte de la ciudadanía.

\subsection{El proyecto "Museion: Quién dónde qué" - Museo MUSEION, Bolzano}

El proyecto del MUSEION de Bolzano, en el norte de Italia, está destinado a jóvenes entre los 11 y 19 años. Los estudiantes están invitados a un recorrido que se desarrolla en una hora y media a través del área expositiva del laboratorio didáctico, y en algunos casos de los almacenes. Los mediadores empiezan mostrando las fotos de los distintos profesionales que trabajan en el museo, explicando sus funciones, y posteriormente asignando un ejercicio en el cual los estudiantes pueden realizar algunas funciones básicas de los profesionales del museo. A partir de esta nueva perspectiva, los jóvenes están posteriormente encargados de diseñar un folleto en el cual promover un evento inventado por ellos que será organizado en el museo. El proyecto tiene varios aspectos en común con el proyecto del MAXXI, pero los desarrolla de una forma distinta, más sintética. El proyecto explora la identidad de la institución Museo, sus funciones, las profesiones relacionadas con la institución y sus características. El objetivo principal es el de acercar los jóvenes al backstage del museo, enseñando el trabajo de las distintas figuras profesionales, visitando el museo a través de una perspectiva diferente Según el testigo de una mediadora del museo, a través de esta actividad los estudiantes están muy interesados por aprender las profesiones museísticas, y despues de la activitidad en algunos casos se sienten tan motivados que declaran su interés por empezar su carrera en el museo. 


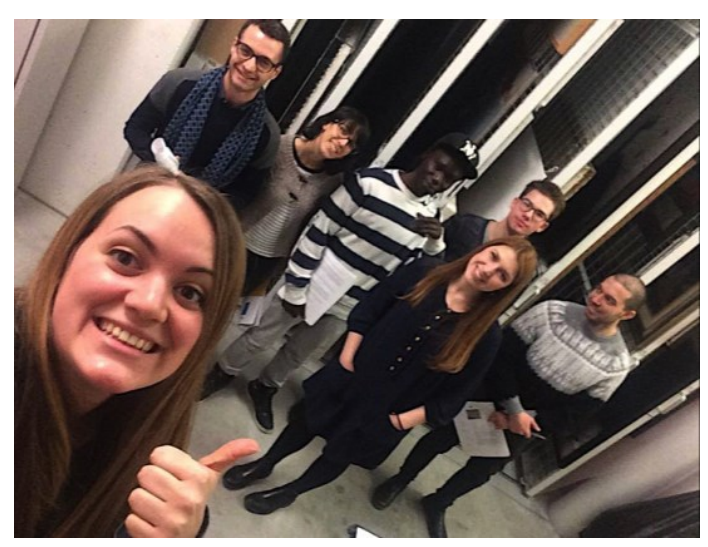

Fig. 4 Jóvenes participantes del proyecto educativo "Museion: Quien donde qué”. @Roberta Pedrini

\section{Conclusiones}

Aunque los tres proyectos en cuestión presentan algunos aspectos comunes que corresponden a la línea de investigación sobre la interdependencia entre conservación y educación en los museos de arte contemporáneo, muestran también discrepancias que subrayan cómo su potencial podría ser desarrollado ulteriormente. Las similitudes entre el proyecto del MAXXI y el del MUSEION son evidentes, pero queda claro cómo en la experiencia de Bolzano los estudiantes no tienen la posibilidad de ser realmente protagonistas, y la brevedad de la actividad impide que se construya una experiencia que deje realmente un rastro en el recorrido educativo de los estudiantes. En cualquier caso, quedan con una nueva perspectiva general sobre el museo, que es útil para construir su identidad de visitantes y ciudadanos corresponsables del destino de sus colecciones contemporáneas. Por otro lado, en el proyecto del MUSEION la conexión con aspectos explícitamente relacionados con la conservación de las obras es débil, así como la colaboración directa con otros departamentos. En el MAXXI en cambio, existía la posibilidad de insistir mucho sobre aspectos conservativos, pero, según el testimonio de los colegas entrevistados, estos aspectos no han sido muy documentados durante la implementación del proyecto. Por ejemplo, los cuadernos en los cuales los estudiantes tenían que anotar diariamente el estado de conservación de las obras no fueron guardados, y ya no están disponibles. Los aspectos del proyecto que han sido guardados son otros, que parecen más relacionados con el objetivo de atraer a los jóvenes al museo en cuanto institución, más que propiamente a sus colecciones. En el curso de las entrevistas han surgido elementos interesantes que evidenciaban la tendencia a motivar la conexión del público con la institución antes que con la colección; en este sentido, algunos estudiantes de El Museo va a la Escuela se han convertido en visitantes habituales del museo, llevando también a sus 
familias, y manteniendo un dialogo constante con los profesionales del museo con los cuales habían colaborado.

La colaboración entre departamentos experimentada en el proyecto del MAXXI, sin muchas consecuencias en prácticas de trabajo posteriores, es en cambio uno de los exitosos elementos del proyecto Doppio Senso del Guggenheim. Aquí las obras usadas en las actividades de educación han sido seleccionadas por el conservador y la curadora del proyecto, los cuales comparten objetivos, conocimientos y experiencias. La propuesta de interacción táctil con las obras ofrece un enfoque completamente nuevo para las personas videntes guiadas por las personas ciegas, poniendo las obras y la colección en el centro de la atención, por delante de la institución. El límite, y al mismo tiempo la potencialidad del proyecto, reside en la dificultad de ser aplicado rápidamente en forma más extensa. Se trata de una experiencia única, que funciona muy bien en grupos pequeños y necesita de tiempo. Al mismo tiempo impone un cambio de perspectiva tanto en los profesionales del museo, como en los visitantes, que están invitados a concentrarse en pocas obras seleccionadas. Este enfoque quizás limita una percepción general sobre la colección, al centrarse tanto en las particularidades de las obras. A partir de la fusión de algunos elementos de este proyecto con los del MAXXI y del MUSEION quizás sería interesante crear un programa de entrenamiento colectivo enfocado en la interacción táctil con las obras, sin renunciar a la perspectiva sobre la colección y las funciones del museo. El camino hacia una real innovación museística que estimule en el publico un sentimiento de apropiación y pertenencia de las colecciones, parecido al que se tiene hacia el patrimonio arquitectónico es largo, pero estos proyectos ponen las semillas necesarias para el desarrollo de nuevas propuestas.

\section{Referencias}

Bilotta d'Onofrio, S.(2016). Il museo fuori dal museo: quando le collezioni raggiungono il territorio. En Branchesi, L. Curzi, V. Mandarano, N., Comunicare il museo oggi. Dalle scelte museologiche al digitale (pp.251-262). Milano: Skira.

Candlin, F. (2004) "Don't Touch! Hands off! Art, blindness and the conservation of expertise" en Pye, E. The power of touch, Left Coast Press, Walnut Creek, CA, United States of America, 2007.

Hooper-Greenhill,E. (1994). The Educational Role of the Museum. London-New York: Routledge.

Huhtamo, E. (2015) "Museums, Interactivity, and the task of Exhibition Anthropology" en Henning, M., The International Handbooks of Museum Studies: Museum Media, First Edition Oxford : Wiley Blackwell.

Llamas Pacheco, R. (2014). Arte Contemporáneo y Restauración. O cómo investigar entre lo material, lo esencial y lo simbólico. Madrid: Editorial Tecnos. 